\title{
Hepatocellular carcinoma development in diabetic patients: a nationwide survey in Japan
}

\author{
Ryosuke Tateishi ${ }^{1}$ (D) Takeshi Matsumura ${ }^{2} \cdot$ Takeshi Okanoue $^{3} \cdot$ Toshihide Shima $^{3}$. \\ Koji Uchino $^{1}$ - Naoto Fujiwara ${ }^{1}$. Takafumi Senokuchi ${ }^{2} \cdot$ Kazuyoshi Kon $^{4}$ • \\ Takayoshi Sasako $^{5,17}$ • Makiko Taniai ${ }^{6}$ - Takumi Kawaguchi ${ }^{7} \cdot$ Hiroshi Inoue $^{8}$. \\ Hirotaka Watada $^{9}$. Naoto Kubota ${ }^{5}$ Hitoshi Shimano ${ }^{10}$. Shuichi Kaneko ${ }^{11}$. \\ Etsuko Hashimoto $^{6} \cdot$ Sumio Watanabe $^{4}$ - Goshi Shiota ${ }^{12} \cdot$ Kohjiro Ueki $^{13}$. \\ Kosuke Kashiwabara $^{14}$ - Yutaka Matsuyama ${ }^{14}$. Hideo Tanaka ${ }^{15}$ - Masato Kasuga ${ }^{16}$. \\ Eiichi Araki ${ }^{2} \cdot K^{2}$ azuhiko Koike ${ }^{1} \cdot$ for the LUCID study investigators
}

Received: 9 July 2020/Accepted: 18 November 2020/Published online: 11 January 2021

(C) The Author(s) 2021

\begin{abstract}
Background Although type 2 diabetes mellitus (T2DM) is a known risk factor for hepatocellular carcinoma (HCC) development, the annual incidence in diabetes patients is far below the threshold of efficient surveillance. This study aimed to elucidate the risk factors for HCC in diabetic patients and to determine the best criteria to identify surveillance candidates.
\end{abstract}

The members of the for the LUCID study investigators are mentioned "Acknowledgments section".

Ryosuke Tateishi

tateishi-tky@umin.ac.jp

1 Department of Gastroenterology, Graduate School of Medicine, The University of Tokyo, 7-3-1 Hongo, Bunkyoku, Tokyo 113-8655, Japan

2 Department of Metabolic Medicine, Kumamoto University Faculty of Life Sciences, Kumamoto, Japan

3 Department of Gastroenterology and Hepatology, Saiseikai Suita Hospital, Suita, Japan

4 Department of Gastroenterology, Juntendo University School of Medicine, Tokyo, Japan

5 Department of Diabetes and Metabolic Diseases, The University of Tokyo Graduate School of Medicine, Tokyo, Japan

6 Institute of Gastroenterology, Department of Internal Medicine, Tokyo Women's Medical University, Tokyo, Japan

7 Division of Gastroenterology, Department of Medicine, Kurume University School of Medicine, Kurume, Japan

8 Metabolism and Nutrition Research Unit, Kanazawa University Institute for Frontier Science Initiative, Kanazawa, Japan
Methods The study included 239 patients with T2DM who were diagnosed with non-viral HCC between 2010 and 2015 , with $\geq 5$ years of follow-up at diabetes clinics of 81 teaching hospitals in Japan before HCC diagnosis, and 3277 non-HCC T2DM patients from a prospective cohort study, as controls. Clinical data at the time of and 5 years before HCC diagnosis were collected.

Results The mean patient age at $\mathrm{HCC}$ diagnosis was approximately 73 years, and $80 \%$ of the patients were male. The proportion of patients with insulin use increased, whereas the body mass index (BMI), proportion of patients with fatty liver, fasting glucose levels, and hemoglobin

9 Department of Medicine, Metabolism and Endocrinology, Juntendo University School of Medicine, Tokyo, Japan

10 Department of Internal Medicine, Metabolism and Endocrinology, Tsukuba University, Tsukuba, Japan

11 Department of Gastroenterology, Kanazawa University Graduate School of Medical Science, Kanazawa, Japan

12 Division of Molecular and Genetic Medicine, Institute of Regenerative Medicine and Biofunction, Graduate School of Medicine, Tottori University, Yonago, Japan

13 Diabetes Research Center, Research Institute, National Center for Global Health and Medicine, Tokyo, Japan

14 Department of Biostatistics, The University of Tokyo Graduate School of Medicine, Tokyo, Japan

15 Fujiidera Public Health Center, Fujiidera, Japan

16 The Institute for Adult Diseases, Asahi Life Foundation, Tokyo, Japan

17 Department of Molecular Sciences on Diabetes, Graduate School of Medicine, The University of Tokyo, Tokyo, Japan 
A1c $(\mathrm{HbA1c})$ levels decreased significantly in 5 years. In the cohort study, 18 patients developed HCC during the mean follow-up period of 4.7 years with an annual incidence of $0.11 \%$. Multivariate logistic regression analyses showed that the FIB-4 index was an outstanding predictor of HCC development along with male sex, presence of hypertension, lower HbA1c and albumin levels, and higher BMI and gamma-glutamyl transpeptidase levels. Receiveroperating characteristic analyses showed that a FIB-4 cutoff value of 3.61 could help identify high-risk patients, with a corresponding annual HCC incidence rate of $1.1 \%$. Conclusion A simple calculation of the FIB-4 index in diabetes clinics can be the first step toward surveillance of HCC with a non-viral etiology.

Keywords Hepatocellular carcinoma - Type 2 diabetes mellitus · FIB-4 index

\section{Introduction}

Globally, liver cancer is the seventh most common cancer and the fourth most common cause of death [1]. In hepatocellular carcinoma (HCC), which accounts for $70 \%$ to $85 \%$ of all liver cancers [2], chronic viral hepatitis plays a major role; approximately $77 \%$ of liver cancer cases were estimated to be attributable to either hepatitis B virus (HBV) or hepatitis C virus (HCV) infection in 2008 [3].

While HCC is a typical example of virus-related cancer, it is also strongly related to lifestyle. Chronic alcoholism is a classical risk factor [4]. Growing evidence suggests that obesity and diabetes increase various cancer risks [5-9], and the liver is one of the organs upon which obesity and obesity-associated conditions have the largest impact $[5,8,10,11]$. Obesity and diabetes represent the largest risk factors for liver cancer development in the United States [12], and the increase in the incidence of HCC was the highest among all types of cancers between 2003 and 2012 [13].

Due to changes in dietary habits in the last four decades, the proportion of overweight males has continued to increase for all ages and in elderly females in Japan. The increase in the overweight and obese population has resulted in an increased number of patients with diabetes mellitus and fatty liver [14, 15]. The Japan Diabetes Society (JDS) conducted a nationwide survey investigating causes of death in 45,708 diseased Japanese patients with diabetes from 2001-2010 [16]. According to the report, the most frequent cause of death was malignant neoplasia (38.3\%), followed by infections (17.0\%), and then vascular diseases (14.9\%). The liver was the second commonest site of cancer-related death after the lung in Japanese diabetic patients, whereas it ranked fifth in the general Japanese population [17]. We also reported that the incidence of HCC with a non-viral etiology increased rapidly between 1991 and 2015 in Japan from a nationwide survey including more than 7000 patients with non-viral etiology [18]. In that report, we concluded that the increase in the rate of obesity among Japanese males over the last three decades has contributed to the increase based on the larger proportion of obese and diabetic patients in the cohort.

International guidelines for $\mathrm{HCC}$ recommend surveillance for HCC in cirrhotic patients [19-21]. However, the precise definition of at-risk population is still controversial. Generally, the definition should be based on the incidence of HCC and potential therapeutic options to be applied. Some reports suggested that an annual incidence of 1.5-2.0\% would warrant the cost-effectiveness of HCC surveillance. According to a large-scale case-control study using the Surveillance Epidemiology and End-Results Program (SEER) database, diabetes carries a threefold increase in the risk of $\mathrm{HCC}$, which is much lower than the 24-fold increased risk of $\mathrm{HBV}$ and $\mathrm{HCV}$ [20, 22]. The estimated annual incidence of HCC development in diabetic patients is less than $0.1 \%$ [23], far below the threshold of efficient surveillance. To elucidate the risk factors for HCC in Japanese diabetic patients and provide the best criteria for surveillance candidates, we conducted a nationwide survey.

\section{Patients and methods}

\section{Study protocol}

This study was conducted as a collaborative project of Japan Society of Hepatology (JSH) and JDS to elucidate the actual profile of hepatocellular carcinoma in diabetes clinics (LUCID). An invitation letter for study participation was sent to 333 hospitals that were certified as education hospitals by both JSH and JDS. The study protocol was approved by the University of Tokyo Medical Research Center Ethics Committee (approval number 11336) and the Institutional Review Board or Ethics Committee of each participating institution. The requirement for informed consent was waived because of the retrospective design. This study was registered with the University Hospital Medical Information Network (UMIN) Clinical Trial Registry (UMIN000026246).

\section{Patients with $\mathrm{HCC}$ in diabetes clinics}

Patients with type 2 diabetes mellitus (T2DM) were eligible if they were initially diagnosed with $\mathrm{HCC}$ at participating hospitals between 2010 and 2015, were negative for both hepatitis B surface antigen (HBsAg) and anti-HCV 
antibody, and had been followed up at a diabetes clinic at the same hospital for at least 5 years before the diagnosis of HCC. HCC was diagnosed pathologically or using imaging criteria on the basis of the Japanese Clinical Practice Guidelines. Hyperattenuation during the arterial phase with washout during the late phase on dynamic CT or dynamic MRI images was regarded as a specific finding [24].

\section{Data collection}

The patients were registered via an electronic data capture system designed by the investigators. We collected data at two points: at the initial diagnosis of $\mathrm{HCC}$ and 5 years before the diagnosis of HCC (Fig. 1). The following patient characteristics were collected: age, sex, body height, body weight, daily alcohol consumption, calendar year of the initial diagnosis of diabetes, type of diabetes, family history of diabetes, medical comorbidities including hypertension, dyslipidemia, and fatty liver, diabetic complications, laboratory data, tumor characteristics, and treatment modalities. The body mass index (BMI), ChildTurcotte-Pugh (CTP) score [25], aspartate aminotransferase (AST) to platelet ratio index (APRI) [26], and fibrosis-4 (FIB-4) index [27] were calculated automatically using the obtained data. The formulae for APRI and FIB-4 were as follows.

$$
\begin{aligned}
& \mathrm{APRI}=\frac{\operatorname{AST}(/ \mathrm{ULN})}{\text { Platelet } \operatorname{count}\left(10^{9} / L\right)} \times 100 \\
& \mathrm{FIB}-4=\frac{\operatorname{Age}(y) \times \operatorname{AST}(U / L)}{\text { Platelet } \operatorname{count}\left(10^{9} / L\right) \times \sqrt{\operatorname{ALT}(U / L)}}
\end{aligned}
$$

\section{Cohort study}

We utilized a pre-existing Japanese nationwide cohort of diabetic patients that enrolled patients with T2DM who visited diabetes clinics at nine participating hospitals from 2008 to 2009. A follow-up survey was conducted in 2015 to collect data at 5 years, including information regarding
HCC development. The detailed protocol of the study was described elsewhere [28, 29].

We retrieved data from the database by excluding the following patients: those who did not have follow-up data including vital status, missing values in age, or with chronic hepatitis B or C. From April 1, 2012, JDS officially started to use the National Glycohemoglobin Standardization Program (NGSP) value of glycated hemoglobin (HbA1c) instead of the original JDS value. As the HbA1c value in the cohort study was obtained using the JDS value, the value was converted according to the following formula.

$\mathrm{HbA} 1 \mathrm{c} \%(\mathrm{NGSP})=1.02 \times \mathrm{HBA} 1 \mathrm{c} \%(\mathrm{JDS})+0.25 \%$

\section{Statistical analysis}

Data were expressed as mean \pm standard deviation when they were approximately normally distributed or otherwise as median with 25 th-75th percentiles for continuous variables and numbers with percentages for categorical variables.

The Student $t$ test or the Wilcoxon rank-sum test was used for comparisons between two continuous variables. For paired continuous variables, the paired Student's $t$ test or the Wilcoxon signed-rank test was used. Differences in the distribution at two time points were assessed using McNemar's test. A logistic regression was used to assess the risk factors for HCC development. We used a receiver operating characteristic (ROC) curve for the assessment of sensitivity, specificity, and likelihood ratio for the positive results of the FIB-4 index. Estimated 5-year HCC rates according to FIB-4 index values were calculated on the basis of Bayes' theorem using annual HCC incidence from the cohort study.

All statistical analyses were performed using SAS software (version 9.4, SAS Institute, Cary, NC) or R software (ver. 3.2.3; R Development Core Team, Vienna, Austria). All tests were two-sided, and $P$ values $<0.05$ were considered to indicate statistical significance.
Fig. 1 Data collection. Patients are enrolled if they were followed-up at the same diabetes clinic for at least 5 years. Data at the time of diagnosis and 5 years before the initial diagnosis of HCC are collected. HCC, hepatocellular carcinoma
Data at two time points were collected
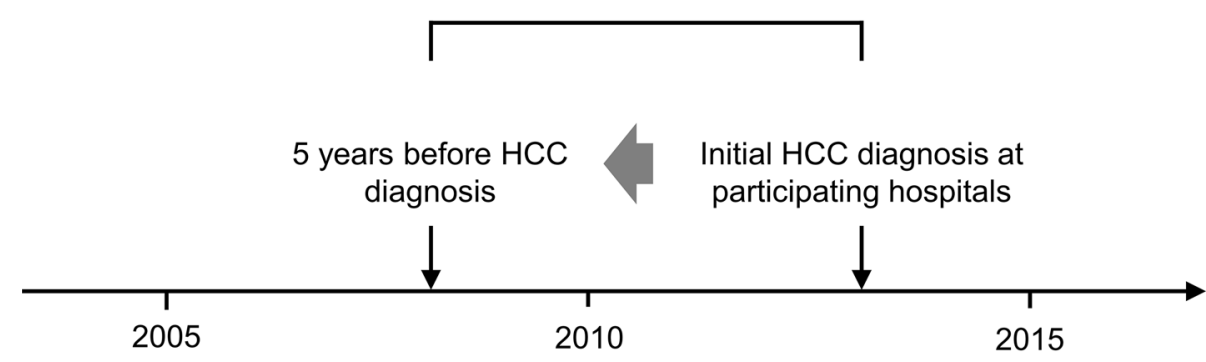

Patients were followed up at diabetes clinics 


\section{Results}

\section{HCC patient profile}

From 272 diabetic patients who were diagnosed at 81 participating hospitals during the study period, we enrolled 239 by excluding those with type 1 diabetes $(N=3)$, missing values for the exact date of the initial and last visits and HCC diagnosis $(N=7)$, unknown duration of diabetes $(N=6)$, hepatitis $C$ status $(N=4)$, fasting glucose $(N=3)$, tumor size or number $(N=7)$, and presence of vascular invasion and extrahepatic metastasis $(\mathrm{N}=3)$ (Appendix Fig. 5).

Baseline characteristics of the HCC patients with T2DM 5 years before and at the time of HCC diagnosis are shown in Table 1. The mean patient age at HCC diagnosis was approximately 73 years, and $80 \%$ of the patients were male. The median interval between the initial diagnosis of diabetes and HCC was 12 years. During the follow-up period of 5 years, the proportion of patients with insulin use increased while BMI and the proportion of patients with fatty liver, fasting glucose, and HbAlc decreased significantly.

Regarding the tumor characteristics, the median size of the main tumor was $3.5 \mathrm{~cm}$, and approximately $60 \%$ of patients had a single tumor. A total of 123 patients (51.0\%) were diagnosed within the Milan criteria (single tumor not larger than $5 \mathrm{~cm}$ or three or fewer tumors, none of which exceeded $3 \mathrm{~cm}$ without vascular invasion or extrahepatic metastasis) [30]. In 224 patients in whom the Child-Pugh class could be calculated, 176 (78.6\%), 39 (17.4\%), and nine $(4.0 \%)$ were categorized as class $\mathrm{A}, \mathrm{B}$, and $\mathrm{C}$, respectively. As a consequence, 130 patients (54.4\%) underwent surgical resection or ablation.

\section{Cohort study}

From 5,642 diabetic patients registered in the database, we enrolled 3295 after excluding type 1 diabetes mellitus $(N=36)$, those without follow-up data $(N=1598)$, with chronic hepatitis B or C $(N=217)$, with previous HCC history at enrollment $(N=39)$, and with missing values for age, exact date of initial and the last visits, and HCC status at the last visit $(N=457)$ (Appendix Fig. 6).

During the mean follow-up period of 4.7 years, 18 patients developed HCC (Fig. 2). The annual HCC incidence calculated by the person-year method was $0.11 \%$ [95\% confidence interval (CI), 0.068-0.181\%]. The baseline characteristics of patients who developed HCC were similar to that of HCC patients in the current study (Table 2).

\section{Risk factors for HCC development}

The HCC patients from the current study and those from the cohort study were combined after excluding overlapping cases $(N=4)$ (Appendix Fig. 7). Logistic regression analysis was performed to elucidate the risk factors for HCC development.

In univariate analyses, the following factors were associated with an increased risk of development of HCC: male sex, old age, higher BMI, AST, ALT, GGT, FIB-4 and APRI, lower platelet count, HbAlc, and albumin, and the presence of hypertension. To avoid multicollinearity, we evaluated several multivariate models. The results from the final model are shown in Fig. 3. Other tested models are shown in Appendix Table 4. Male sex, presence of hypertension, lower HbAlc and albumin, and higher GGT and FIB-4 were significant predictors of HCC development in diabetic patients. The presence of dyslipidemia was a negative risk factor with marginal significance.

\section{ROC analysis}

Since the FIB-4 index at entry showed a remarkable impact on HCC development, we performed an $a d-h o c$ analysis to evaluate various cut-off values for FIB-4 and sensitivity, specificity, and positive likelihood ratio for the HCC incidence using ROC analysis (Fig. 4). When a cut-off value of 2.67 was applied, which was recommended as the best indicator for advanced fibrosis [31], the sensitivity was approximately $50 \%$. We found that the cut-off value of 3.61 maximized the positive likelihood ratio, and the corresponding annual $\mathrm{HCC}$ incidence rate was estimated at $1.1 \%$ (Table 3). The result was robust after excluding those with a history of heavy alcohol drinking ( $\geq 60 \mathrm{~g} /$ day) (Appendix Fig. 8).

\section{Discussion}

Diabetes is recognized as a well-established risk factor for HCC, however, other risk factors in diabetes patients without viral hepatitis have rarely been investigated so far because of the low incidence rate in the population [32]. In this study, we elucidated various potential risk factors for HCC, including male sex, old age, higher BMI, and higher FIB-4. The FIB-4 index, in particular, showed outstanding ability for extracting a high-risk group for surveillance.

Among various mechanisms that have been suggested as involved in the link between diabetes and hepatocarcinogenesis, insulin resistance and subsequent hyperinsulinemia, a key driver in metabolic syndrome, play a major role [33]. Nonalcoholic fatty liver disease (NAFLD), known as a hepatic manifestation of metabolic syndrome, and its 
Table 1 Patient characteristics 5 years before and at the time of HCC diagnosis $(N=239)$

\begin{tabular}{|c|c|c|c|}
\hline & Five years before HCC diagnosis & At the time of HCC diagnosis & $P$ \\
\hline Follow-up period, years & $5.00 \pm 0.33$ & - & \\
\hline Age, years & $67.8 \pm 8.8$ & $72.8 \pm 8.8$ & \\
\hline Male, $n(\%)$ & $191(79.9)$ & - & \\
\hline Diabetes duration, years & $7(3-14)$ & $12(8-19)$ & \\
\hline Diabetic complications, $n(\%)$ & $117(49.0)$ & - & \\
\hline \multicolumn{4}{|l|}{ Alcohol consumption, g/day } \\
\hline$\leq 20, n(\%)$ & $159(66.5)$ & - & \\
\hline$>20$ and $<60, n(\%)$ & $41(17.2)$ & - & \\
\hline$\geq 60, n(\%)$ & $39(16.3)$ & - & \\
\hline BMI, $\mathrm{kg} / \mathrm{m}^{2}$ & $26.0 \pm 4.5$ & $25.5 \pm 4.8$ & 0.01 \\
\hline Diabetes treatment & & & 0.001 \\
\hline Diet only, $n(\%)$ & $23(9.6)$ & $18(7.5)$ & \\
\hline Oral antidiabetic, $n(\%)$ & $145(60.7)$ & $133(55.6)$ & \\
\hline Insulin, $n(\%)$ & $71(29.7)$ & $88(36.8)$ & \\
\hline Diabetes complications, $n(\%)$ & $117(49.0)$ & - & \\
\hline Hypertension, $n(\%)$ & $168(70.3)$ & $174(72.3)$ & 0.32 \\
\hline Dyslipidemia, $n(\%)$ & 109 (45.6) & $111(46.4)$ & 0.87 \\
\hline Fatty liver, $n(\%)^{\mathrm{a}}$ & $108(60.3)$ & $110(51.2)$ & 0.03 \\
\hline Fasting glucose, $\mathrm{mg} / \mathrm{dL}$ & $143(121-181)$ & $132(110-173)$ & 0.02 \\
\hline $\mathrm{HbA1c}, \%$ & $7.1(6.5-8.1)$ & $6.9(6.3-7.6)$ & $<0.0001$ \\
\hline Total cholesterol, mg/dL & $181.1 \pm 34.8$ & - & \\
\hline Albumin, g/dL & $4.07 \pm 0.41$ & $3.83 \pm 0.57$ & $<0.0001$ \\
\hline $\mathrm{AST}, \mathrm{U} / \mathrm{L}^{\mathrm{b}}$ & $36(25-46)$ & $37(26-52)$ & 0.10 \\
\hline $\mathrm{ALT}, \mathrm{U} / \mathrm{L}^{\mathrm{c}}$ & $34(22-47)$ & $26(19-41)$ & 0.001 \\
\hline $\mathrm{GGT}, \mathrm{U} / \mathrm{L}^{\mathrm{d}}$ & $60(34-142)$ & $83(45-160)$ & 0.08 \\
\hline Creatinine, $\mathrm{mg} / \mathrm{dL}^{\mathrm{e}}$ & $0.80(0.68-0.98)$ & $0.87(0.71-1.13)$ & $<0.0001$ \\
\hline Hemoglobin, $\mathrm{mg} / \mathrm{dL}^{\mathrm{f}}$ & $13.9 \pm 1.8$ & $12.7 \pm 2.2$ & $<0.0001$ \\
\hline Platelet count, $10^{4} / \mu \mathrm{L}$ & $15.5(11.7-19.6)$ & $14.6(10.1-20.0)$ & 0.17 \\
\hline $\mathrm{APRI}^{\mathrm{g}}$ & $0.62(0.38-1.10)$ & $0.73(0.41-1.28)$ & 0.008 \\
\hline FIB- $4^{h}$ & $2.73(1.90-4.30)$ & $3.74(2.43-5.76)$ & $<0.0001$ \\
\hline \multicolumn{4}{|l|}{ HCC characteristics } \\
\hline Maximal tumor size, $\mathrm{cm}$ & - & $3.5(2.0-6.5)$ & \\
\hline Maximal tumor $\leq 3 \mathrm{~cm}, n(\%)$ & - & $110(46.0)$ & \\
\hline Single tumor, $n(\%)$ & - & $141(59.0)$ & \\
\hline Vascular invasion, $n(\%)$ & - & $36(15.1)$ & \\
\hline Extrahepatic metastasis, $n(\%)$ & - & $20(8.4)$ & \\
\hline Within Milan criteria & & $123(51.0)$ & \\
\hline $\mathrm{AFP} \geq 20 \mathrm{ng} / \mathrm{mL}, n(\%)$ & - & $84(36.1)$ & \\
\hline $\mathrm{DCP} \geq 100 \mathrm{mAU} / \mathrm{mL}, n(\%)^{\mathrm{h}}$ & - & $120(53.3)$ & \\
\hline \multicolumn{4}{|l|}{ HCC treatment } \\
\hline Resection, $n(\%)$ & - & $62(25.9)$ & \\
\hline Ablation, $n(\%)$ & - & $68(28.5)$ & \\
\hline TACE, $n(\%)$ & - & $117(49.0)$ & \\
\hline Systemic therapy, $n(\%)$ & - & $8(3.3)$ & \\
\hline Radiation therapy, $n(\%)$ & - & $3(1.3)$ & \\
\hline Supportive care, $n(\%)$ & & $24(10.0)$ & \\
\hline
\end{tabular}

Data are expressed as mean \pm standard deviation when approximately normally distributed or otherwise median (25th-75th percentiles) for continuous variables and number (percentage) for categorical variables

Data are missing for ${ }^{\mathrm{a}} 60,{ }^{\mathrm{b}} 7,{ }^{\mathrm{c}} 7,{ }^{\mathrm{d}} 19,{ }^{\mathrm{e}} 8,{ }^{\mathrm{f}} 9,{ }^{\mathrm{g}} 12$, and ${ }^{\mathrm{h}} 13$ patients 5 years before HCC diagnosis and ${ }^{\mathrm{a}} 24,{ }^{\mathrm{d}} 5$, and ${ }^{\mathrm{h}} 4$ patients at HCC diagnosis

$A S T$ aspartate aminotransferase; $A L T$ alanine aminotransferase; $A F P$ alpha-fetoprotein; $B M I$ body mass index; $D C P$ des-gamma-carboxy prothrombin; FIB-4 fibrosis-4; GGT gamma-glutamyl transpeptidase; APRI AST to platelet ratio index; HbAlc hemoglobin A1c; TACE transarterial chemoembolization 


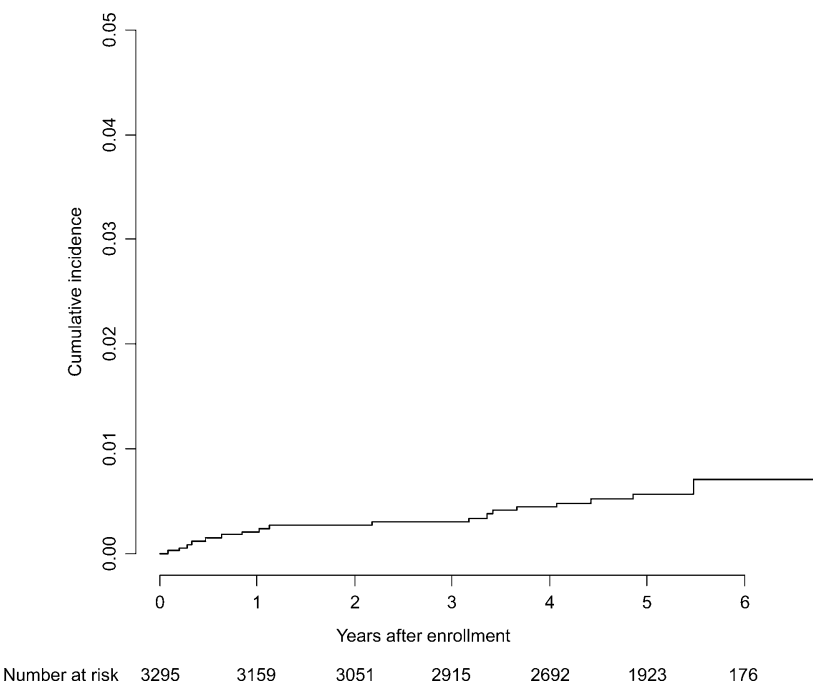

Fig. 2 Kaplan-Meier estimate of cumulative incidence of hepatocellular carcinoma in the cohort study progressive form, nonalcoholic steatohepatitis (NASH), are evident background conditions of HCC development [32]. However, the HCC incidence rate in NAFLD or NASH patients is as low as less than 0.1 per year unless the patients progress to cirrhosis [34-36]. Therefore, to identify patients with advanced fibrosis is mandatory for the surveillance of non-viral HCC. Besides, hepatic steatosis decreases according to disease progression, and in the final stage of the disease, known as burned-out NASH, fatty liver cannot be detected by regular ultrasonography [37]. The proportion of patients with fatty liver decreased during the follow-up period of this study. Therefore, the presence of fatty liver is not necessary for the enrollment of surveillance.

The FIB-4 index was originally developed for the prediction of significant fibrosis in patients with HIV/HCV coinfection [27]. Now, it is widely used for prediction of various liver diseases, including NAFLD [31]. Since the
Table 2 Characteristics of patients in the cohort study

\begin{tabular}{|c|c|c|c|}
\hline & $\begin{array}{l}\mathrm{HCC} \\
N=18\end{array}$ & $\begin{array}{l}\text { non-HCC } \\
N=3277\end{array}$ & $P$ \\
\hline Age at entry, years & $69.72 \pm 10.3$ & $63.3 \pm 12.2$ & 0.02 \\
\hline Male, $n(\%)$ & $13(72.2)$ & 1887 (57.6) & 0.21 \\
\hline Alcohol consumption, g/day ${ }^{\mathrm{a}}$ & & & $<0.0001$ \\
\hline$\leq 20, n(\%)$ & $9(50.0)$ & $2307(80.5)$ & \\
\hline$>20$ and $<60, n(\%)$ & $4(22.2)$ & $433(15.1)$ & \\
\hline$\geq 60, n(\%)$ & $5(27.8)$ & $126(4.4)$ & \\
\hline BMI, $\mathrm{kg} / \mathrm{m}^{2}$ & $25.9(22.5-27.0)$ & $24.0(21.6-26.9)$ & 0.25 \\
\hline Diabetes treatment ${ }^{\mathrm{b}}$ & & & 0.80 \\
\hline Diet only, $n(\%)$ & $4(22.2)$ & $520(16.5)$ & \\
\hline Oral antidiabetic, $n(\%)$ & $9(50.0)$ & $1626(51.7)$ & \\
\hline Insulin, $n(\%)$ & $5(27.8)$ & $999(31.8)$ & \\
\hline Hypertension, $n(\%)$ & $11(61.1)$ & $1680(51.3)$ & 0.40 \\
\hline Dyslipidemia, $n(\%)$ & $6(33.3)$ & 1594 (48.6) & 0.29 \\
\hline Fasting glucose, $\mathrm{mg} / \mathrm{dL}$ & $121(112-138)$ & $144(119-184)$ & 0.03 \\
\hline $\mathrm{HbA} 1 \mathrm{c}, \%$ & $6.5(5.75-7.35)$ & $7.4(6.70-8.40)$ & 0.007 \\
\hline Total cholesterol, $\mathrm{mg} / \mathrm{dL}^{\mathrm{c}}$ & $163.4 \pm 35.1$ & $194.5 \pm 39.7$ & 0.003 \\
\hline Albumin, $g / \mathrm{dL}^{\mathrm{d}}$ & $3.77 \pm 0.40$ & $4.21 \pm 0.41$ & 0.0003 \\
\hline $\mathrm{AST}, \mathrm{U} / \mathrm{L}^{\mathrm{e}}$ & $36.5(25-46)$ & $21(18-28)$ & $<0.0001$ \\
\hline $\mathrm{ALT}, \mathrm{U} / \mathrm{L}^{\mathrm{f}}$ & $33.5(22-53)$ & $21(15-31)$ & 0.009 \\
\hline GGT, U/L $L^{\mathrm{g}}$ & $89.5(36-173)$ & $27(18-47)$ & 0.001 \\
\hline Platelet count, $10^{4} / \mu \mathrm{L}^{\mathrm{h}}$ & $18.3(10.1-19.9)$ & $21.3(17.7-25.0)$ & $<0.0001$ \\
\hline $\mathrm{APRI}^{\mathrm{i}}$ & $0.534(0.450-0.474)$ & $0.275(0.204-0.383)$ & $<0.0001$ \\
\hline FIB $-4^{\mathrm{j}}$ & $2.33(1.02-4.74)$ & $1.47(1.04-2.03)$ & $<0.0001$ \\
\hline
\end{tabular}

Data are expressed as mean \pm standard deviation when approximately normally distributed or otherwise median (25th-75th percentiles) for continuous variables and number (percentage) for categorical variables Data are missing for ${ }^{\mathrm{c}} 2,{ }^{\mathrm{d}} 1,{ }^{\mathrm{g}} 2$, and ${ }^{\mathrm{h}} 12$ patients with $\mathrm{HCC}$, and ${ }^{\mathrm{a}} 412,{ }^{\mathrm{b}} 132,{ }^{\mathrm{c}} 268,{ }^{\mathrm{d}} 324,{ }^{\mathrm{e}} 31,{ }^{\mathrm{f}} 30,{ }^{\mathrm{g}} 101,{ }^{\mathrm{h}} 153$, ${ }^{\mathrm{i}} 158$, and ${ }^{\mathrm{j}} 160$ patients without HCC

$H C C$ hepatocellular carcinoma; AST aspartate aminotransferase; $A L T$ alanine aminotransferase; BMI body mass index; GGT gamma-glutamyl transpeptidase; APRI AST to platelet ratio index; HbAlc hemoglobin A1c 


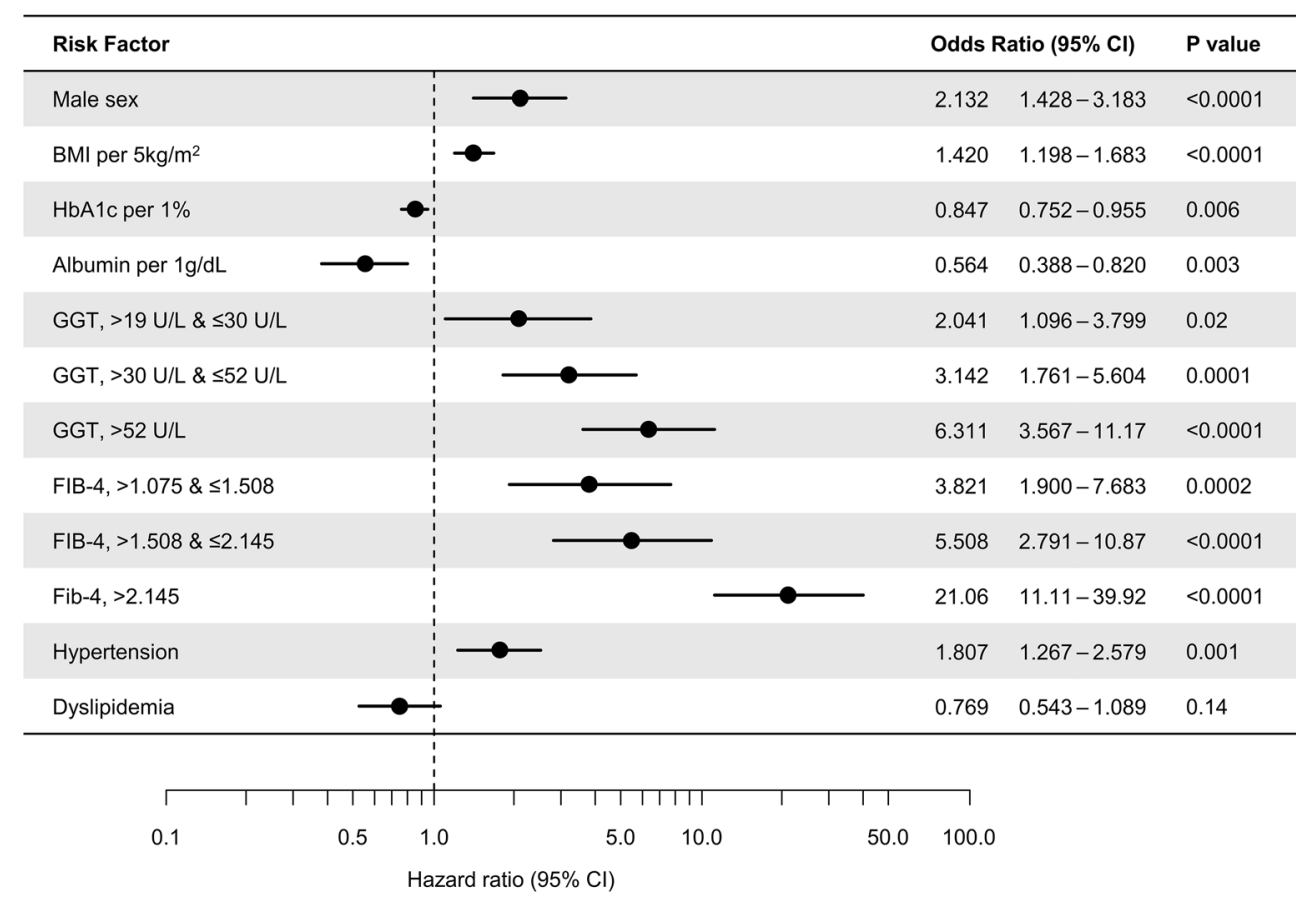

Fig. 3 Multivariate logistic regression for HCC development (final model). The closed circles indicate point estimates of hazard ratios. The horizontal bars indicate $95 \%$ confidence interval. HCC, hepatocellular carcinoma

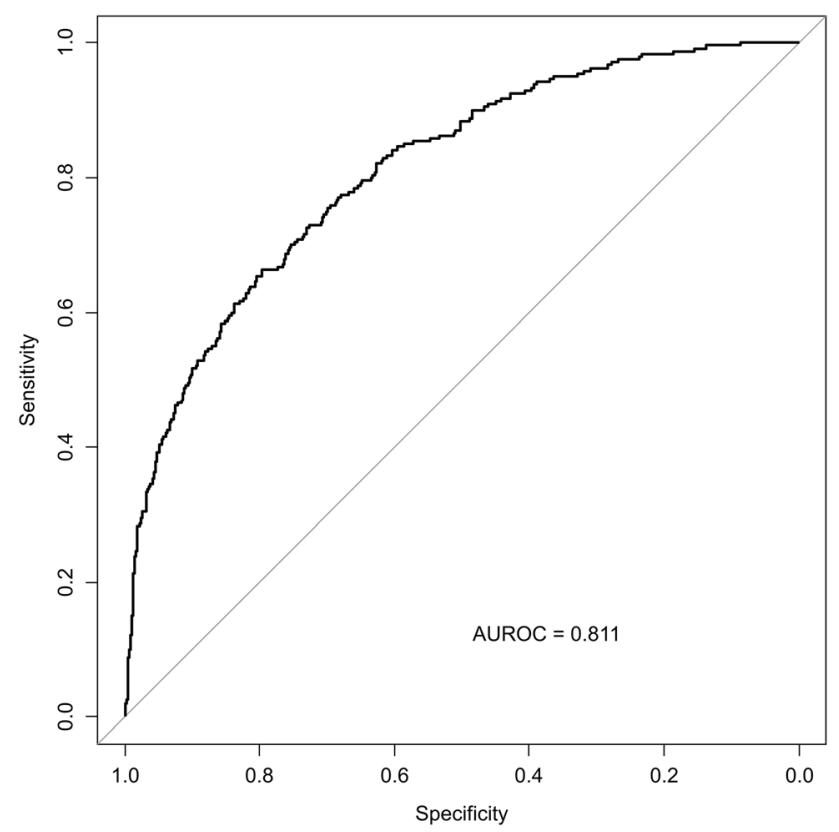

Fig. 4 Receiver-operating characteristics (ROC) curve of FIB-4 for HCC development in diabetic patients. FIB-4, fibrosis-4; HCC, hepatocellular carcinoma

FIB-4 index includes age, there can be an argument that it overestimates liver fibrosis in elderly patients [38]. However, this disadvantage of the FIB-4 index in fibrosis estimation is rather an advantage in the risk estimation for HCC because old age is a strong risk factor in hepatocarcinogenesis. The APRI, another commonly used index for liver fibrosis, showed similar but slightly smaller predictive power than the FIB-4 index since the former does not include age as a component.

Although the specific protocol of surveillance for HCC in diabetes patients is open to debate, it would be a good idea to use the FIB-4 index as an entry test. Once-a-year examination of AST, ALT, and platelet count is inexpensive and acceptable. The cut-off value of 3.61 may identify patients at a $1 \%$ annual risk of HCC development. However, since the cut-off value misses approximately twothirds of HCC patients, a two-step screening strategy combining FIB-4 and other non-invasive tests would be better. For that purpose, the cut-off value of 2.67 is a promising candidate. If the cut-off value of 2.67 was applied, approximately $10 \%$ of patients in the T2DM cohort would have undergone further examination.

In this study, higher BMI indicated an increased risk of HCC even after adjustment for other risk factors. Obesity, a key component of metabolic syndrome, causes adipose tissue remodeling and induces increased secretion of proinflammatory adipokines, including TNF- $\alpha$ and IL-6, and decreased anti-inflammatory adipokines like adiponectin, which potentially accelerates hepatocarcinogenesis $[39,40]$. Obesity is reported to be a risk factor for HCC in both hepatitis B and C [41, 42].

Among key components of metabolic syndrome, whilst hypertension was an independent risk factor for $\mathrm{HCC}$ in this study, dyslipidemia was inversely related to $\mathrm{HCC}$ 
Table 3 Sensitivity, specificity, and positive likelihood ratio of annual HCC development according to cut-off values of FIB-4 index

\begin{tabular}{lllcl}
\hline FIB-4 & Sensitivity & Specificity & Positive likelihood ratio & Estimated annual HCC incidence (\%) \\
\hline 1.00 & 0.983 & 0.229 & 1.27 & 0.14 \\
1.50 & 0.863 & 0.521 & 1.80 & 0.20 \\
2.00 & 0.708 & 0.737 & 2.69 & 0.30 \\
2.50 & 0.546 & 0.874 & 4.34 & 0.50 \\
2.67 & 0.517 & 0.898 & 5.07 & 0.60 \\
3.00 & 0.421 & 0.937 & 6.67 & 0.80 \\
3.50 & 0.342 & 0.963 & 9.21 & 1.0 \\
3.61 & 0.333 & 0.968 & 10.3 & 1.1 \\
3.70 & 0.304 & 0.970 & 9.98 & 1.1 \\
4.00 & 0.283 & 0.979 & 13.2 & 1.4 \\
5.00 & 0.186 & 0.987 & 15.0 & 1.6 \\
\hline
\end{tabular}

FIB-4, fibrosis-4; HCC, hepatocellular carcinoma development, probably because of reduced cholesterol synthesis in advanced liver diseases. It should be noted that the absence of dyslipidemia along with decreased hepatic steatosis may not indicate a lower risk of HCC. Similarly, HbA1c superficially improved during the observation period of 5 years. This was mainly because the hemoglobin level also decreased due to the shortened half-life of erythrocytes according to cirrhosis progression. Thus, improved $\mathrm{HbA} 1 \mathrm{c}$ values may also not indicate a decreased risk of HCC.

Excessive alcohol intake is an established risk for HCC [43]. In the present study, $16.3 \%$ of HCC patients and $27.8 \%$ of patients in the T2DM cohort who develop HCC were categorized as heavy drinkers ( $\geq 60 \mathrm{~g} /$ day), whereas the proportion was $4.4 \%$ in non-HCC patients. It is controversial whether heavy alcohol drinkers with T2DM should be treated within the same surveillance strategy for non-drinkers and moderate drinkers or outside the framework as a definite category of alcoholic liver disease. There is a concern that the FIB-4 index may not be useful for alcoholic liver disease in which AST elevation is more prominent than NAFLD. However, our previous reports of a nation-wide survey of non-viral HCC showed that the FIB-4 index was stable compared to AST and GGT during moderation in drinking [18].

The present study has several limitations. First, since the number of HCC cases in the cohort study was only 18 , the annual incidence of HCC may not be statistically accurate. The cut-off points of the FIB-4 index, which was based on the incidence, should be validated in a prospective study. Second, this study could not elucidate the effect of antidiabetic treatment on hepatocarcinogenesis. Metformin reportedly reduces the risk of $\mathrm{HCC}$ in diabetic patients [44]. We collected data regarding anti-diabetic treatment in this study. However, as metformin was regarded as contraindicated for cirrhosis and treatment choice for anti- diabetic drugs varies according to years, the statistical power was not enough to obtain a robust result. Third, as the HCC patients can be regarded as cancer-free for 5 years ahead of HCC diagnosis according to the enrollment criteria, the magnitude of risk factors for HCC development may be underestimated. Nevertheless, the risk factors that remained after multivariate analysis would be invariant. Forth, the prospective cohort consisted only of patients who were followed at the tertiary care hospitals. Since those who were referred to other affiliated hospitals and thus excluded from the prospective study might have a better condition, the cumulative HCC incidence could be overestimated.

In conclusion, we found various common risk factors for HCC development in diabetic patients. A simple calculation of the FIB-4 index in diabetes clinics can be the first step for the surveillance of HCC with non-viral etiology. We would start a prospective study to validate the efficacy of FIB-4-based surveillance strategy in the near future.

Acknowledgements The following investigators enrolled patients in the LUCID study: Yoshiyasu Karino, Shuhei Hige, Masatomo Sekiguchi (Hokkaido P.W.F.A.C. Sapporo-Kosei General Hospital), Koji Ogawa, Hideaki Miyoshi, KyuYong Cho (Hokkaido University Hospital), Masaru Baba, Atsushi Inoue (Japan Community Health Care Organization Hokkaido Hospital), Kazuobu Aso, Mitsuyoshi Okada (Asahikawa Medical University), Yasuhiro Takikawa, Kei Endo, Yasushi Ishigaki, Hirobumi Togashi (Iwate Medical University), Michiaki Unno, Takanori Morikawa, Hideki Katagiri, Shojiro Sawada (Tohoku University Hospital), Hiromasa Ohira, Atsushi Takahashi, Michio Shimabukuro, Akihiro Kudo (Fukushima Medical University Hospital), Naomi Tanaka, Junko Mitsuhashi, Toshifumi Tokai (Koyama Memorial Hospital), Yasushi Matsuzaki, Tadashi Ikegami (Tokyo Medical University Ibaraki Medical Center), Masato Imai (Utsunomiya Memorial Hospital), Kou Nishikawa, Sadao Takahashi (Ageo Central General Hospital), Sumiko Nagoshi, Kosuke Maezawa, Masafumi Matsuda (Saitama Medical Center), Tetsuhiro Chiba, Masayuki Yokoyama, Koutaro Yokote, Yoko Hidetaka (Chiba University Hospital), Mitsuhiko Moriyama, Hitomi Nakamura, Midori Fujishiro (Nihon University Hospital), Tadakazu Hisamatsu, Kaori Nishikawa (Kyorin University Hospital), Toshihiko 
Nouchi, Yuki Sakurai, Harumi Daikoku (Showa General Hospital), Michinori Murayama (Self-Defence Forces Central Hospital), Yoshinori Saigusa, Takashi Matsui (Toho Umiversity Medical Center Ohashi Hospital), Kazuhiko Koike, Ryosuke Tateishi, Naoto Kubota, Takayoshi Sasako (The University of Tokyo Hospital), Hidenari Nagai, Takanori Mukozu, Takahisa Hirose, Masahiko Miyagi (Toho Umiversity Medical Center Omori Hospital), Sumio Watanabe, Kazuyoshi Kon, Hirotaka Watada, Hiroaki Sato (Juntendo University Hospital), Akira Mizuki, Masanori Miura (Keiyu Hospital), Yuzuru Sato, Rena Kaneko, Kumiko Hamano, Hiroko Kato (Japan Organization of Occupational Health and Safety Kanto Rosai Hospital), Kentaro Kikuchi, Yusuke Kajiyama (Teikyo University Hospital, Mizonokuchi), Makoto Chuma, Hiroko Ito (Yokohama City University Medical Center), Hiroshi Yasuda, Nobuyuki Matsumoto, Yasushi Tanaka, Yoshio Nagai (St. Marianna University School of Medicine Hospital), Kento Imajo, Yasuo Terauchi, Yuzuru Ito (Yokohama City University Hospital), Shuji Terai, Hirohito Sone, Satoshi Matsunaga (Niigata University Medical \& Dental Hospital), Kiyoshi Furuta (Matsumoto Medical Center), Eiji Tanaka, Takeji Umemura, Mitsuhisa Komatsu, Yoshihiko Sato (Shinshu University Hospital), Yoshinobu Hinoue, Akiko Shimizu (Toyama City Hospital ), Terumi Takahara, Kengo Kawai, Kazuyuki Tobe, Isao Usui (Toyama University Hospital), Hajime Ohta, Takuya Komura, Seiichiro Kurita (National Hospital Organization Kanazawa Medical Center), Nobuyuki Toshikuni, Daisuke Koya, Ai Watanabe (Kanazawa Medical University Hospital), Yasunari Nakamoto, Tomoyuki Nemoto, Tadashi Konoshita (University of Fukui Hospital), Yoshimasa Kobayashi, Shin Shimoyama (Hamamatsu University Hospital), Daisaku Nishimura (Toyota Kosei Hospital ), Masami Imoto, Sayori Nakashima, Tatsuo Mizuno (Kariya Toyota General Hospital), Kentaro Yoshioka, Naoto Kawabe, Suzuki Atsushi, Ayako Kakita (Fujita Health University Hospital), Yuji Kojima, Kazuya Murata (Japanese Red Cross Ise Hospital), Yoshiyuki Takei, Motoo Iwasa, Yutaka Yano (Mie University Hospital), Masahiko Kondo, Motozumi Okamoto (Japanese Red Cross Otsu Hospital), Hiroyuki Kimura (Japanese Red Cross Kyoto Daiichi Hospital), Hiroyuki Marusawa, Yuji Eso, Yoshihito Fujita (Kyoto University Hospital), Hideki Fujii, Masaaki Inaba, Masanori Emoto (Osaka City University Hospital), Takeshi Kurose (Kansai Electric Power Hospital), Takeshi Okanoue, Toshihide Shima, Shinichi Mogami (Saiseikai Suita Hospital), Rinako Tsuda, Emiko Nomura (Kansai Medical University Medical Center), Masayoshi Ohnishi (Osaka General Hospital of West Japan Railway Company), Masatoshi Kudo, Naoshi Nishida, Hiroshi Ikegami, Hiroyuki Ito (Kindai University Hospital), Hirokazu Oyamada, Yasutaka Nagao, Hiroshi Okada (Matsushita Memorial Hospital), Masahide Oshita, Tetsuyuki Yasuda (Osaka Police Hospital), Toshifumi Ito, Nobuyuki Tatsumi (Japan Community Health care Organization Osaka Hospital), Teruhisa Morikawa (Akashi City Hospital), Noriko Takahara (Ako City Hospital), Takumi Fukumoto, Motofumi Tanaka, Wataru Ogawa, Yushi Hirota (Kobe University Hospital), Seitetsu Yung, Keiji Iida (Hyogo Prefectural Kakogawa Medical Center), Hitoshi Yoshiji, Naotaka Shimozato (Nara Medical University
Hospital), Gen Inoue, Tomonao Hirobata (Japanese Red Cross Wakayama Medical Center), Junichi Okano, Tsuyoshi Okura (Tottori University Hospital ), Yasushi Uchida, Toshiaki Sato (Matsue Red Cross Hospital), Miwa Kawanaka, Takatoshi Anno (Kawasaki Medical School General Medical Center), Keisuke Hino, Yasuyuki Tomiyama, Hideaki Kaneto (Kawasaki Medical School Hospital), Shinichi Fujioka, Eri Sasaki, Tatsuaki Nakato (Okayama Saiseikai General Hospital), Keiji Tsuji, Yohji Honda, Nozomu Kamei, Mitsue Miyahara (Hiroshima Red Cross Hospital \& Atomic-bomb Survivors Hospital), Tsutomu Masaki, Kyoko Oura (Kagawa University Hospital), Yoshiyasu Kisaka, Shozo Miyauchi, Isao Makino (Uwajima City Hospital), Yoichi Hiasa, Yohei Koizumi, Osawa Haruhiko, Toshimi Hadate (Ehime University Hospital), Takeaki Sato, Isao Ichino (National Hospital Organization Kokura Medical Center), Hideyuki Nomura, Nobuyuki Yamashita (Shin-Kokura Hospital), Hiroshi Maeno, Sunao Matubayashi, (Fukuoka Tokushukai Hospital), Kenta Motomura, Yukie Ishibashi, Makoto Ide, Yoko Moribe (Aso Iizuka Hospital ), Masaru Harada, Michihiko Shibata, Yosuke Okada, Keiichi Torimoto (Hospital of University of Occupational and Environmental Health, Japan), Takuji Torimura, Takumi Kawaguchi, Yuji Tajiri (Kurume University Hospital), Nobuhiko Higashi, Sakae Nohara (Steel Memorial Yawata Hospital), Yasunori Kawaguchi, Toru Yoshimura (Saga-ken Medical Centre Koseikan), Keizo Anzai, Shinji Iwane, (Saga University Hospital), Kazuhiko Nakao, Naota Taura, Norio Abiru, Yoshitaka Mori (Nagasaki University Hospital), Masataka Seike, Mizuki Endo (Oita University Hospital), Tadanobu Kuribayashi (Koga General Hospital).

Open Access This article is licensed under a Creative Commons Attribution 4.0 International License, which permits use, sharing, adaptation, distribution and reproduction in any medium or format, as long as you give appropriate credit to the original author(s) and the source, provide a link to the Creative Commons licence, and indicate if changes were made. The images or other third party material in this article are included in the article's Creative Commons licence, unless indicated otherwise in a credit line to the material. If material is not included in the article's Creative Commons licence and your intended use is not permitted by statutory regulation or exceeds the permitted use, you will need to obtain permission directly from the copyright holder. To view a copy of this licence, visit http://creativecommons. org/licenses/by/4.0/.

Funding This research was supported by AMED under Grant Number JP17ek0210065, JP17fk0210304, and JP20fk0210040.

\section{Appendix}

See Figs. 5, 6, 7, 8 and Table 4. 
Fig. 5 Patient enrollment flow: hepatocellular carcinoma patients at diabetes clinics

Fig. 6 Patient enrollment flow: cohort study of diabetic patients
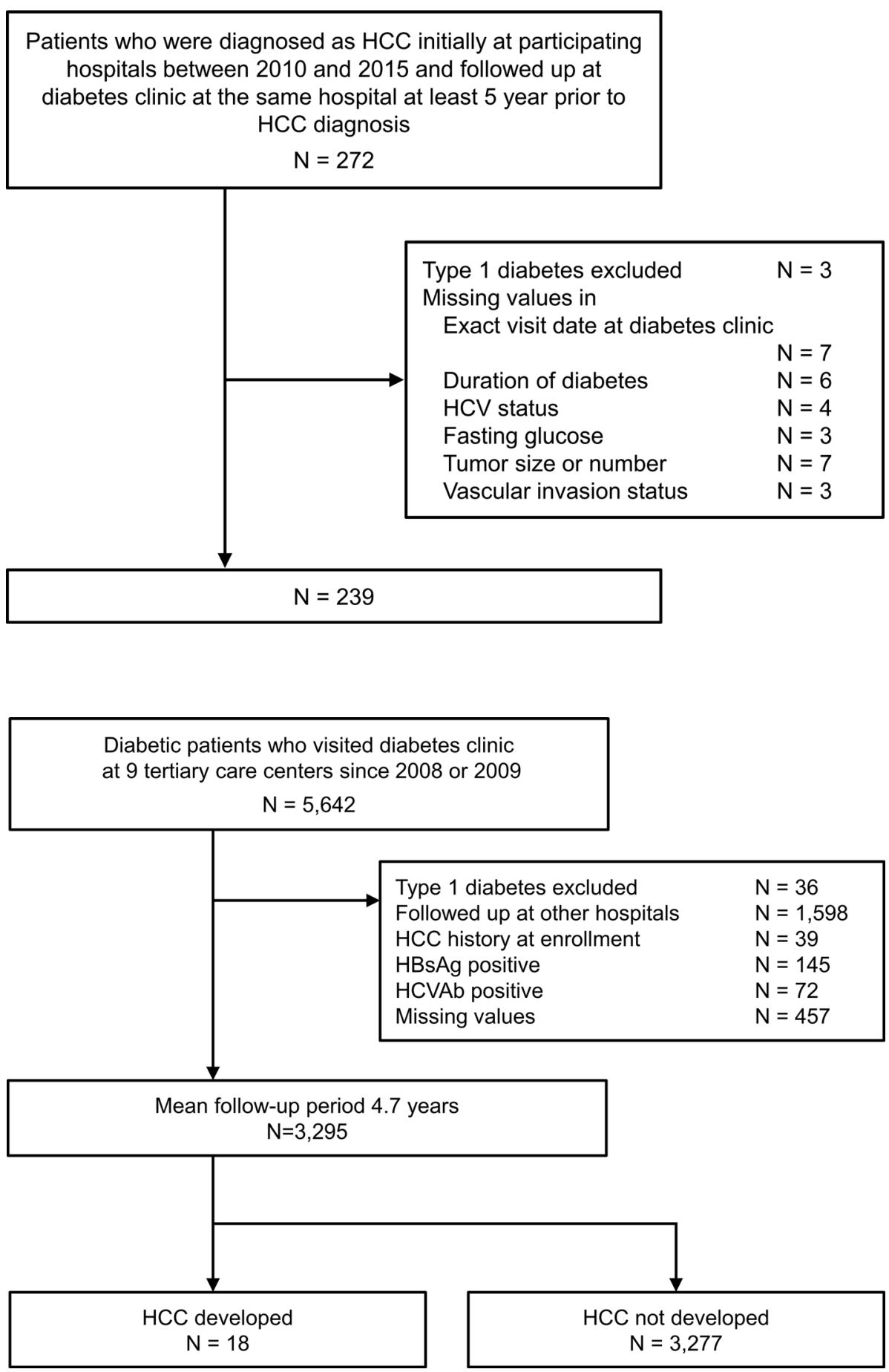
Fig. 7 Patient enrollment: HCC cases and non-HCC cases were compared. * Overlapping cases $(N=4)$ were excluded

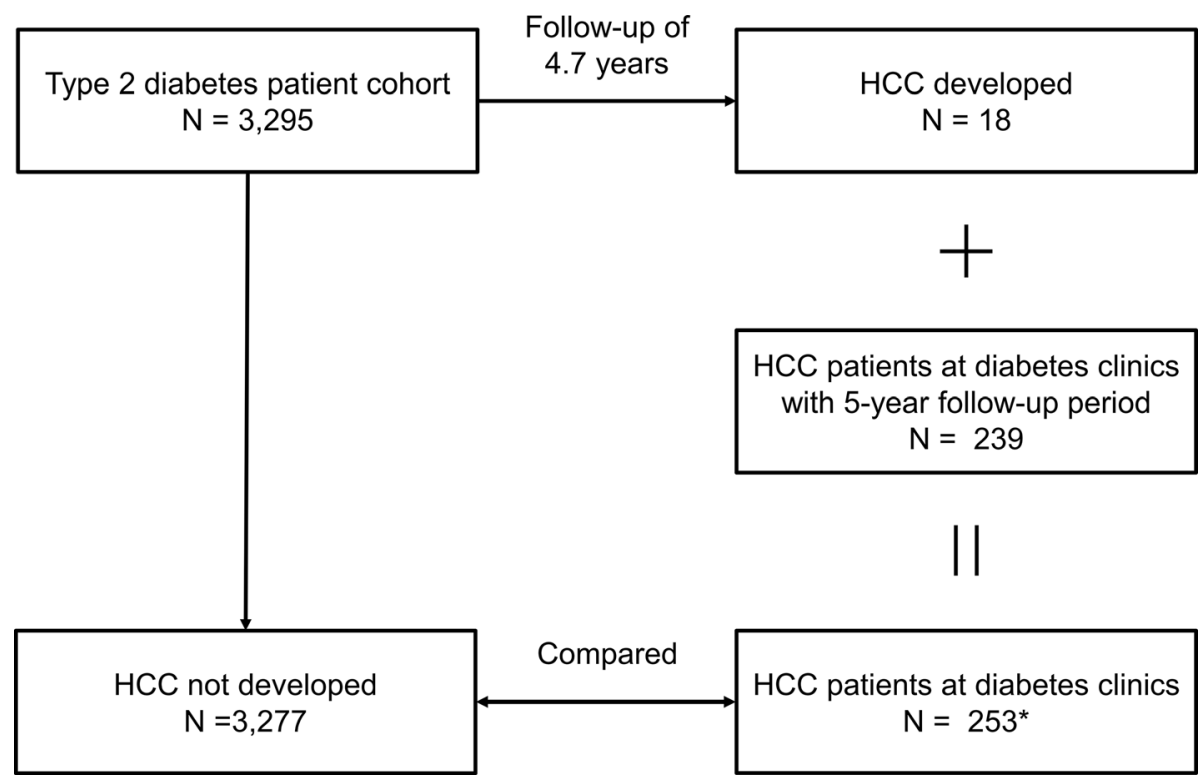

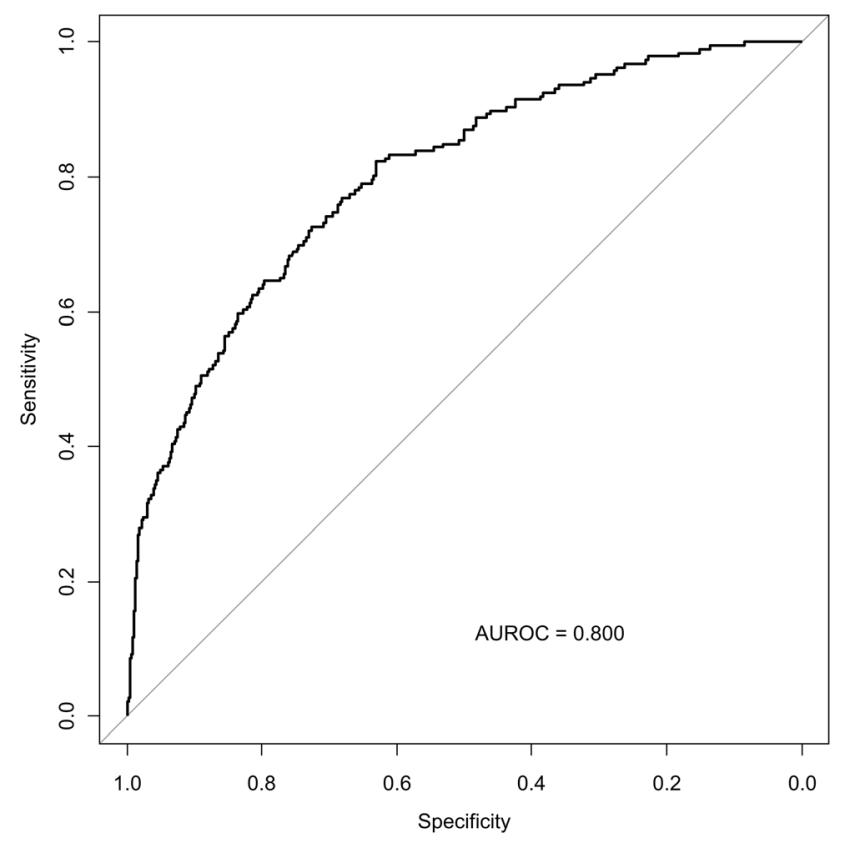

Fig. 8 Receiver-operating characteristics (ROC) curve of fibrosis-4 for hepatocellular carcinoma: The subgroup analysis excludes those with a history of alcohol intake $\geq 60 \mathrm{~g} /$ day 
Table 4 Univariate and multivariate logistic regression analyses

\begin{tabular}{|c|c|c|c|c|c|c|c|c|c|}
\hline \multirow[b]{2}{*}{ Variables } & \multicolumn{3}{|c|}{ Univariate analysis } & \multicolumn{3}{|c|}{ Multivariate analysis: model 1} & \multicolumn{3}{|c|}{ Multivariate analysis: model 2} \\
\hline & $\begin{array}{l}\text { Odds } \\
\text { ratio }\end{array}$ & $\begin{array}{l}95 \% \text { confidence } \\
\text { interval }\end{array}$ & & $\begin{array}{l}\text { Odds } \\
\text { ratio }\end{array}$ & $\begin{array}{l}95 \% \text { confidence } \\
\text { interval }\end{array}$ & & $\begin{array}{l}\text { Odds } \\
\text { ratio }\end{array}$ & $\begin{array}{l}95 \% \text { confidence } \\
\text { interval }\end{array}$ & \\
\hline Male vs. female & 2.50 & $(1.75,3.56)$ & $<0.0001$ & 2.07 & $(1.36,3.14)$ & 0.0006 & 2.40 & $(1.59,3.62)$ & $<0.0001$ \\
\hline Age, per 10 years & 1.38 & $(1.20,1.60)$ & $<0.0001$ & 1.48 & $(1.22,1.79)$ & $<0.0001$ & 1.57 & $(1.31,1.88)$ & $<0.0001$ \\
\hline BMI, per $5 \mathrm{~kg} / \mathrm{m}^{2}$ & 1.31 & $(1.14,1.50)$ & 0.0001 & 1.40 & $(1.16,1.68)$ & 0.0004 & 1.34 & $(1.13,1.59)$ & 0.0009 \\
\hline AST, IU/mL & 1.03 & $(1.03,1.04)$ & $<0.0001$ & 1.02 & $(1.00,1.03)$ & 0.007 & & & \\
\hline ALT, IU/mL & 1.01 & $(1.01,1.02)$ & $<0.0001$ & 1.00 & $(0.99,1.01)$ & 0.524 & & & \\
\hline $\begin{array}{l}\text { Platelet count, } \\
10^{4} / \mu \mathrm{L}\end{array}$ & 0.82 & $(0.79,0.84)$ & $<0.0001$ & 0.85 & $(0.82,0.88)$ & $<0.0001$ & & & \\
\hline $\mathrm{HbA} 1 \mathrm{c}, \%$ & 0.86 & $(0.78,0.96)$ & 0.009 & 0.85 & $(0.75,0.96)$ & 0.008 & 0.83 & $(0.74,0.94)$ & 0.003 \\
\hline Albumin, $\mathrm{g} / \mathrm{dL}$ & 0.43 & $(0.32,0.59)$ & $<0.0001$ & 0.58 & $(0.39,0.86)$ & 0.007 & 0.45 & $(0.31,0.66)$ & $<0.0001$ \\
\hline GGT, U/L ${ }^{\mathrm{a}}$ & & & $<0.0001$ & & & $<0.0001$ & & & $<0.0001$ \\
\hline$\leq 19$ & 1 & & & & & & & & \\
\hline$>19$ and $\leq 30$ & 2.16 & $(1.20,3.92)$ & 0.011 & 1.93 & $(1.04,3.60)$ & 0.038 & 1.77 & $(0.95,3.33)$ & 0.074 \\
\hline$>30 \& \leq 52$ & 4.00 & $(2.32,6.90)$ & $<0.0001$ & 2.91 & $(1.61,5.24)$ & 0.0004 & 2.20 & $(1.22,3.98)$ & 0.009 \\
\hline$>52$ & 10.86 & $(6.43,18.34)$ & $<0.0001$ & 6.41 & $(3.50,11.71)$ & $<0.0001$ & 4.14 & $(2.28,7.51)$ & $<0.0001$ \\
\hline FIB $-4^{\mathrm{a}}$ & & & $<0.0001$ & & & & & & \\
\hline$\leq 1.075$ & 1 & & & & & & & & \\
\hline $\begin{array}{l}>1.075 \\
\text { and } \leq 1.508\end{array}$ & 3.55 & $(1.79,7.04)$ & 0.0003 & & & & & & \\
\hline $\begin{array}{l}>1.508 \\
\& \leq 2.145\end{array}$ & 5.42 & $(2.80,10.49)$ & $<0.0001$ & & & & & & \\
\hline$>2.145$ & 24.89 & $(13.54,45.77)$ & $<0.0001$ & & & & & & \\
\hline $\mathrm{APRI}^{\mathrm{a}}$ & & & $<0.0001$ & & & & & & $<0.0001$ \\
\hline$\leq 0.208$ & 1 & & & & & & & & \\
\hline $\begin{array}{l}>0.208 \\
\text { and } \leq 0.287\end{array}$ & 2.28 & $(1.02,5.10)$ & 0.045 & & & & 1.92 & $(0.84,4.37)$ & 0.120 \\
\hline $\begin{array}{l}>0.287 \\
\text { and } \leq 0.412\end{array}$ & 7.79 & $(3.80,15.98)$ & $<0.0001$ & & & & 5.27 & $(2.51,11.05)$ & $<0.0001$ \\
\hline$>0.412$ & 29.63 & $(14.84,59.16)$ & $<0.0001$ & & & & 17.3 & $(8.36,35.80)$ & $<0.0001$ \\
\hline Hypertension & 2.08 & $(1.51,2.86)$ & $<0.0001$ & 2.08 & $(1.43,3.02)$ & 0.0001 & 1.78 & $(1.25,2.55)$ & 0.002 \\
\hline Dyslipidemia & 0.74 & $(0.55,1.00)$ & 0.053 & 0.84 & $(0.59,1.20)$ & 0.336 & 0.76 & $(0.54,1.08)$ & 0.130 \\
\hline
\end{tabular}

$B M I$ body mass index; GGT gamma-glutamyl transpeptidase; FIB-4 fibrosis-4; APRI aminotransferase to platelet ratio index

${ }^{\mathrm{a}} \mathrm{GGT}$, APRI and FIB-4 were categorized according to the quartile points

\section{References}

1. Bray F, Ferlay J, Soerjomataram I, et al. Global cancer statistics 2018: GLOBOCAN estimates of incidence and mortality worldwide for 36 cancers in 185 countries. Cancer J Clin. 2018;68:394-424.

2. Torre LA, Bray F, Siegel RL, et al. Global cancer statistics, 2012. Cancer J Clin. 2015;65:87-108.

3. de Martel C, Ferlay J, Franceschi S, et al. Global burden of cancers attributable to infections in 2008: a review and synthetic analysis. Lancet Oncol. 2012;13:607-15.

4. Yu MC, Tong MJ, Govindarajan S, et al. Nonviral risk factors for hepatocellular carcinoma in a low-risk population, the nonAsians of Los Angeles County. California J Natl Cancer Inst. 1991;83:1820-6.
5. Calle EE, Rodriguez C, Walker-Thurmond K, et al. Overweight, obesity, and mortality from cancer in a prospectively studied cohort of US adults. N Engl J Med. 2003;348:1625-38.

6. Jee SH, Sull JW, Park J, et al. Body-mass index and mortality in Korean men and women. N Engl J Med. 2006;355:779-87.

7. Bhaskaran K, Douglas I, Forbes H, et al. Body-mass index and risk of 22 specific cancers: a population-based cohort study of 524 million UK adults. Lancet. 2014;384:755-65.

8. Collaboration ERF. Diabetes mellitus, fasting glucose, and risk of cause-specific death. N Engl J Med. 2011;364:829-41.

9. Giovannucci E, Harlan DM, Archer MC, et al. Diabetes and cancer: a consensus report. CA Cancer J Clin. 2010;60:207-21.

10. El-Serag HB, Hampel H, Javadi F. The association between diabetes and hepatocellular carcinoma: a systematic review of epidemiologic evidence. Clin Gastroenterol Hepatol. 2006;4:369-80. 
11. El-Serag HB, Rudolph KL. Hepatocellular carcinoma: epidemiology and molecular carcinogenesis. Gastroenterology. 2007;132:2557-76.

12. Makarova-Rusher OV, Altekruse SF, McNeel TS, et al. Population attributable fractions of risk factors for hepatocellular carcinoma in the United States. Cancer. 2016;122:1757-65.

13. Ryerson AB, Eheman CR, Altekruse SF, et al. Annual Report to the Nation on the Status of Cancer, 1975-2012, featuring the increasing incidence of liver cancer. Cancer. 2016;122:1312-37.

14. Kawamori R. Diabetes trends in Japan. Diabetes Metab Res Rev. 2002;18(Suppl 3):S9-13.

15. Kojima $S$, Watanabe $N$, Numata $M$, et al. Increase in the prevalence of fatty liver in Japan over the past 12 years: analysis of clinical background. J Gastroenterol. 2003;38:954-61.

16. Nakamura J, Kamiya H, Haneda M, et al. Causes of death in Japanese patients with diabetes based on the results of a survey of 45,708 cases during 2001-2010: Report of the Committee on Causes of Death in Diabetes Mellitus. J Diabetes Investig. 2017;8:397-410.

17. Cancer Registry and Statistics [database on the Internet]. 2017. https://ganjoho.jp/reg_stat/statistics/dl/index.html\#mortality.Accessed 9 Jan 2020

18. Tateishi R, Uchino K, Fujiwara N, et al. A nationwide survey on non-B, non-C hepatocellular carcinoma in Japan: 2011-2015 update. J Gastroenterol. 2019;54:367-76.

19. Marrero JA, Kulik LM, Sirlin CB, et al. Diagnosis, Staging, and Management of Hepatocellular Carcinoma: 2018 Practice Guidance by the American Association for the Study of Liver Diseases. Hepatology. 2018;68:723-50.

20. European Association for the Study of the Liver. EASL Clinical Practice guidelines: management of hepatocellular carcinoma. J Hepatol. 2018;69:182-236.

21. Omata M, Cheng AL, Kokudo N, et al. Asia-Pacific clinical practice guidelines on the management of hepatocellular carcinoma: a 2017 update. Hepatol Int. 2017;11:317-70.

22. Davila JA, Morgan RO, Shaib Y, et al. Diabetes increases the risk of hepatocellular carcinoma in the United States: a population based case control study. Gut. 2005;54:533-9.

23. El-Serag HB, Tran T, Everhart JE. Diabetes increases the risk of chronic liver disease and hepatocellular carcinoma. Gastroenterology. 2004;126:460-8.

24. The committee for revision of the Clinical Practice Guidelines for Hepatocellular Carcinoma. Clinical Practice Guidelines for Hepatocellular Carcinoma. The Japan Society of Hepatology 2009 update. Hepatol Res 2010;40:16-47

25. Pugh RN, Murray-Lyon IM, Dawson JL, et al. Transection of the oesophagus for bleeding oesophageal varices. $\mathrm{Br} \mathrm{J}$ Surg. 1973;60:646-9.

26. Wai CT, Greenson JK, Fontana RJ, et al. A simple noninvasive index can predict both significant fibrosis and cirrhosis in patients with chronic hepatitis C. Hepatology. 2003;38:518-26.

27. Sterling RK, Lissen E, Clumeck N, et al. Development of a simple noninvasive index to predict significant fibrosis in patients with HIV/HCV coinfection. Hepatology. 2006;43:1317-25.

28. Shima T, Uto H, Ueki K, et al. Clinicopathological features of liver injury in patients with type 2 diabetes mellitus and comparative study of histologically proven nonalcoholic fatty liver diseases with or without type 2 diabetes mellitus. J Gastroenterol. 2013;48:515-25.

29. Shima T, Uto H, Ueki K, et al. Hepatocellular carcinoma as a leading cause of cancer-related deaths in Japanese type 2 diabetes mellitus patients. J Gastroenterol. 2019;54:64-77.

30. Mazzaferro V, Regalia E, Doci R, et al. Liver transplantation for the treatment of small hepatocellular carcinomas in patients with cirrhosis. N Engl J Med. 1996;334:693-9.

31. Shah AG, Lydecker A, Murray K, et al. Comparison of noninvasive markers of fibrosis in patients with nonalcoholic fatty liver disease. Clin Gastroenterol Hepatol. 2009;7:1104-12.

32. Marengo A, Rosso C, Bugianesi E. Liver cancer: connections with obesity, fatty liver, and cirrhosis. Annu Rev Med. 2016;67:103-17.

33. Karagozian R, Derdak Z, Baffy G. Obesity-associated mechanisms of hepatocarcinogenesis. Metabolism. 2014;63:607-17.

34. Ascha MS, Hanouneh IA, Lopez R, et al. The incidence and risk factors of hepatocellular carcinoma in patients with nonalcoholic steatohepatitis. Hepatology. 2010;51:1972-8.

35. Angulo P, Kleiner DE, Dam-Larsen S, et al. Liver fibrosis, but no other histologic features, is associated with long-term outcomes of patients with nonalcoholic fatty liver disease. Gastroenterology. 2015;149(389-97):e10.

36. Hagstrom H, Nasr P, Ekstedt M, et al. Fibrosis stage but not NASH predicts mortality and time to development of severe liver disease in biopsy-proven NAFLD. J Hepatol. 2017;67:1265-73.

37. Powell EE, Cooksley WG, Hanson R, et al. The natural history of nonalcoholic steatohepatitis: a follow-up study of forty-two patients for up to 21 years. Hepatology. 1990;11:74-80.

38. McPherson S, Hardy T, Dufour JF, et al. Age as a confounding factor for the accurate non-invasive diagnosis of advanced NAFLD fibrosis. Am J Gastroenterol. 2017;112:740-51.

39. Park EJ, Lee JH, Yu GY, et al. Dietary and genetic obesity promote liver inflammation and tumorigenesis by enhancing IL-6 and TNF expression. Cell. 2010;140:197-208.

40. Dalamaga M, Diakopoulos KN, Mantzoros CS. The role of adiponectin in cancer: a review of current evidence. Endocr Rev. 2012;33:547-94.

41. Yu MW, Shih WL, Lin CL, et al. Body-mass index and progression of hepatitis B: a population-based cohort study in men. J Clin Oncol. 2008;26:5576-82.

42. Ohki T, Tateishi R, Sato T, et al. Obesity is an independent risk factor for hepatocellular carcinoma development in chronic hepatitis C patients. Clin Gastroenterol Hepatol. 2008;6:459-64.

43. Morgan TR, Mandayam S, Jamal MM. Alcohol and hepatocellular carcinoma. Gastroenterology. 2004;127:S87-96.

44. Singh S, Singh PP, Singh AG, et al. Anti-diabetic medications and the risk of hepatocellular cancer: a systematic review and meta-analysis. Am J Gastroenterol. 2013;108:881-91.

Publisher's Note Springer Nature remains neutral with regard to jurisdictional claims in published maps and institutional affiliations. 